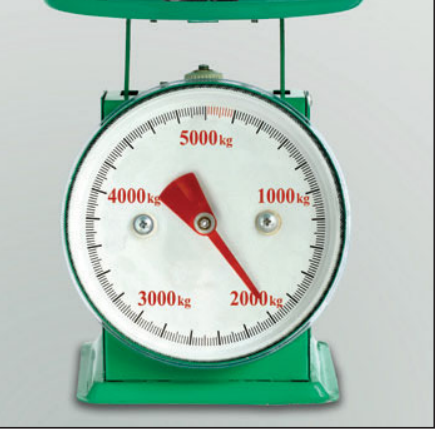

\section{Fuel efficiency drives the auto industry to reduce vehicle weight}

\author{
By Arthur L. Robinson \\ Feature Editors: Alan I. Taub and Gregory A. Keoleian
}

$I^{n}$ $\mathrm{n}$ this age of global warming, the automotive industry is seeking to minimize the energy required to manufacture and operate its products without sacrificing performance and safety or increasing cost. Toward this end, whether cars and trucks are powered by internal-combustion engines or batteries, lowering vehicle weight is a major contributor to reducing energy consumption by increasing fuel efficiency. "The industry is driven by fuel efficiency," said David Matlock of the Colorado School of Mines, who has helped develop advanced high-strength steels (AHSSs) used in autos.

According to Alan Taub of the University of Michigan and former General Motors executive, industry researchers have succeeded in squeezing more weight out of auto materials with a three-pronged strategy: Finding stronger, lighter materials than used previously; developing manufacturing processes for parts made from advanced materials that require minimal retooling; and designing parts that use as little material as possible (a process called topology optimization).

Despite lightweighting advances, however, vehicle weight from 1985 to 2000 actually increased, owing to the addition of vehicle features needed to meet government regulations and consumer preferences. After around 2005, the weight of vehicles leveled off as continuing improvements in weight-reduction technology were used to offset further increases in content. During this era, despite no improvement in weight, fuel economy increased as a result of improved powertrain efficiency.

"Future improvements in fuel efficiency are expected through further lightweighting and vehicle electrification," said Gregory Keoleian, also at the University of Michigan. A useful industry guideline for light vehicles is that a $10 \%$ weight reduction generates a $6 \%$ improvement in fuel economy, or for electric vehicles, a $14 \%$ driving range increase. The numbers may be different, but the same principle holds for other types of vehicles. For example, Keoleian and his colleagues have reported up-to-date and more precise energy savings from lightweighting obtained using physicsbased models for a variety of light-duty and heavy-duty vehicles as well as other transportation modes, such as trains and aircraft.

Going forward, two trends have emerged as the foci for further advances in lightweighting. The first is to build vehicles (body, chassis, and engine) using multiple materials rather than just one, whether steel or a lighter alternative (see Figure). "In addition to weight reduction, combining two or more materials in an optimized design can give better performance than one alone," said polymer researcher Uday Vaidya of the Institute for Advanced Composites Manufacturing Innovation (IACME) and the University of Tennessee. Eventually, each vehicle subsystem would be made from a material selected as the best alternative based on properties, manufacturability, and cost. The second is the use of Integrated Computational Materials Engineering (ICME) to find improved alloy compositions with the required mechanical properties and acceptable cost and the manufacturing processes to make and shape them, as well as to verify their performance in service. Integrated refers to linking the computational techniques needed, which span length scales from atomic to macroscopic.

For much of the 20th century, ferrous materials dominated the structural portions of automobiles-low-carbon steel alloys for the body and cast iron for the engine block - owing to their low cost and ease of forming parts. To reduce vehicle weight, which also offers advantages beyond increased fuel efficiency, such as improved drivability and (for trucks) carrying larger loads, an interest in replacing low-carbon (mild) steel grew toward the end of the century.

Aluminum, an increasingly common alternative material in autos and trucks, can yield a weight savings of $40-60 \%$, with a cost premium of 1.3-2, as compared to cast iron and low-carbon steel. The comparable figures for even lighter magnesium are 60-75\% and 1.5-2.5, according to a 2015 US Department of Energy (DOE) report that also covered AHSSs, titanium, glass fiber composites, and carbon fiber composites (see Table I). Keeping cost down is a major challenge, said Taub. "When one considers the life-cycle cost from extraction of ore to recycling, it turns out that strong, light materials are more expensive, but the need for less material can offset some of their intrinsically higher cost."

Today's vehicles have all of these materials, with steel and aluminum being used most often as the major materials in structural components. Alan Luo of The Ohio State University, who specializes in aluminum and other lightweight metal alloys, pointed to recent models of the Ford F-150 truck. "The truck bodies are aluminum," said Luo, "reducing the vehicle weight by more than $320 \mathrm{~kg}$ and accounting for $13 \%$ of the total vehicle weight." Magnesium and polymer composites are finding increasing use in vehicles, as well. "Polymers and composites now account for $10-15 \%$ of some commercial vehicles and up to $50 \%$ for performance vehicles," said Vaidya. The 2019 GMC Denali now offers a carbon fiber truck bed, for example. 
Mixing materials brings some challenges. While steel alloys are relatively easy to form into the desired shape and the technology is well developed for joining parts by welding, bolting, and riveting, this is not always the case for alloys based on other materials. For example, steel can be made into many shapes by stamping sheets at room temperature, while aluminum may need to be heated. Joining steel and aluminum parts raises another problem. "Steel parts can be readily welded together, and so can parts made from aluminum, but joining steel and aluminum requires more expensive techniques, such as self-pierce riveting," said Luo.

Mixing materials poses a significant challenge to recycling, as well.

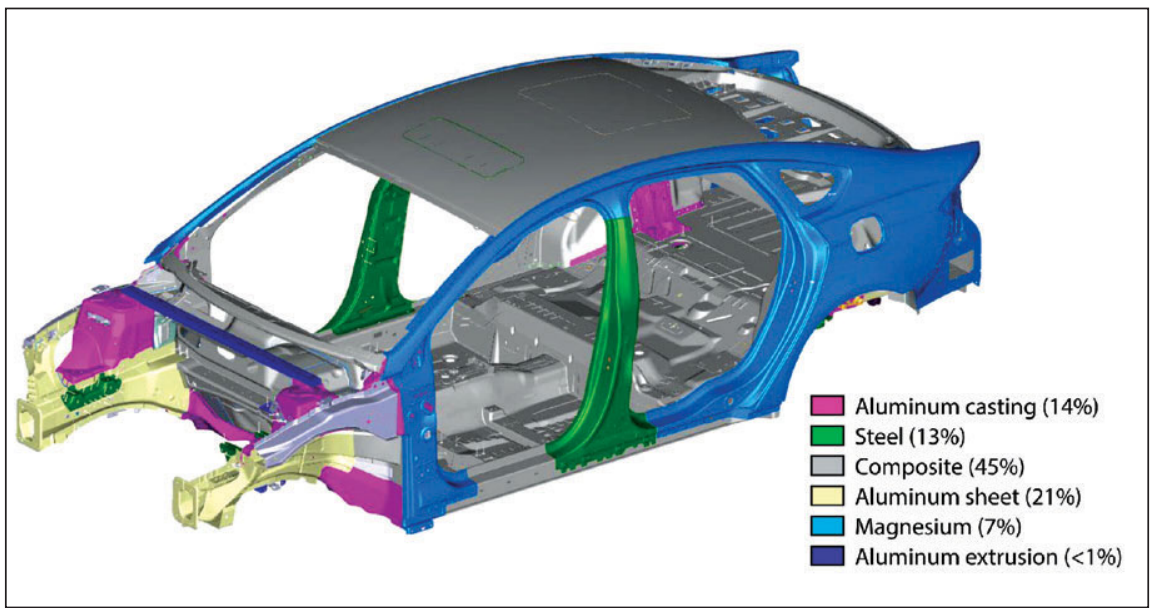

Figure. With the goal of making a lighter-weight vehicle without sacrificing performance and safety or increasing cost, the trend is toward using multiple materials for the various structural subsystems. Here, the mix of materials comprising the "white body" protecting driver and passengers on a demonstration vehicle based on a 2013 Ford Fusion is shown. Image courtesy of A. Taub et al., Annu. Rev. Mater. Res. 49, 327 (2019).
Steel alloys have a long history of recyclability. The same is not so true for lighter materials. "Lightweight materials often have a complicated end-of-life phase, with issues including incomplete recovery, separation challenges, contamination, and lack of a closed-loop recycling path," said Keoleian, who with Taub and other collaborators has enunciated green principles for vehicle lightweighting, with recycling holding a prominent position in more than one of the principles. They cite the example of overcasting (or overmolding for composites), in which two materials are brought into contact to form a reaction zone that creates a continuous bond between the two. This technique has enabled the use of mixed-material construction to reduce the mass of automotive subsystems, but the mixing can present issues at end of life because separating the two materials is not always technically or economically feasible.

Researchers have also found that it takes more than simply mixing a variety of materials. To find out how much weight savings can result from choosing the optimum material for each vehicle subsystem, a DOE-funded project carried out by Ford and Vehma International that ended in 2015 aimed to demonstrate a multimaterial lightweight vehicle based on a 2013 Ford Fusion. The most advanced design made extensive use of magnesium and carbon fiber and aspired to a weight saving of a little more than $50 \%$, as compared to the Fusion, but fell short, owing to materials-performance limitations.

In the search for an alloy with improved performance, materials engineers have traditionally relied on tables listing the properties of the various alloys available. However, in the search for new, lighter, stronger alloys, such tables either may not exist or are incomplete. Usually, lengthy trial-and-error methods investigating many compositions and microstructures are the backup approach to developing new materials.

To see why the process takes so long (10 years or more in the auto industry), consider that an alloy's properties depend not only on a composition characterized by a complex phase diagram with multiphase regions (and sometimes metastable phases). They also rely on microstructural features such as grain size, second-phase precipitates, dislocations and other defect configurations, and texture, all of which can be altered for better or worse during manufacturing processes common in the auto industry, such as casting, thermomechanical processing, and powder consolidation for metals and various thermo-forming approaches for polymer composites. With interest dawning in even more complex materials such as high-entropy alloys, the problem will only grow.

"In today's fast-paced environment," said Gregory Olson of QuesTek Innovations LLC and Northwestern University, "a competitive advantage goes to those who can compress the materials development and concurrent engineering cycle." As an expression of the Materials Genome Initiative, ICME has emerged during the last decade as a fast-growing tool for multiscale modeling of materials, from electronic and atomic structure to microstructures and manufacturing processes, and to macroscopic behavior in service. QuesTek, for example, has based its business on ICME and related computational approaches to develop several new alloys, such as its Ferrium ${ }^{\circledR}$ steels for the aerospace industry.

As for the auto industry, Matlock points to a succession, now in the third generation, of AHSSs developed over the decades. "There have been big advances in steels based on detailed microstructural control at each step, and new products are now on the market supporting a growing industry," said Matlock. A2017 report from the DOE Office of Energy Efficiency \& Renewable Energy by a multi-institutional industrial-academic consortium, including General Motors and Ford, showed how ICME could contribute. According to the report, the project demonstrated the potential for third-generation AHSSs to achieve up to $30 \%$ weight savings in an automotive structure at a cost penalty of only USD\$0.32-\$1.26 per pound of weight saved. The model enables the user to design third-generation steels to desired mechanical properties in terms of strength and ductility. 
Similar progress is seen for lightweight materials, such as aluminum and magnesium. "ICME has a big role now. We can use older techniques like CALPHAD (CALculation of PHAse Diagrams) to predict phases, and ICME simulation tools to predict grain size, precipitates, and so on to optimize strength," said Luo.

But what is the reliability of the ICME predictions? asked Raymond Decker of Thixomat Technologies LLC, which develops magnesium-based materials for consumer electronics, automobiles, and biocompatible devices. "Today, to validate the ICME predictions for materials properties, it is often necessary to make trial heats of the materials and test them, but we still get the end result faster," said Decker. As new data tables are completed and confidence grows, this need should fade.

One way to lighten materials for automobiles does not require new materials. Topology optimization is the term that describes the process of designing parts that minimize the amount of material needed to provide the required performance. In one startling example, simply relocating the bolt holes in an engine block produced a 30\% weight savings. "Topology optimization is primarily a geometrical distribution of material over a given design domain, but independent of materials microstructure," said Luo, "and design software to achieve it is now commercially available." Eventually, though, materials engineers will want to integrate it with ICME techniques for further weight saving and performance improvement.
Table I. Impact of material substitution.

Material Replaced $\quad$ Mass Reduction (\%)

Relative Cost per Part

Mild steel, cast iron

$60-75$

$1.5-2.5$

Mild steel

Mild steel, cast iron

$50-60$

$2-10+$

$40-60$

$1.5-3+$

Mild steel, cast iron

Mild steel

Mild steel

Mild steel
$1.3-2$

$1.5-10+$

$1-1.5$

$1-1.5$

Courtesy of A. Taub et al., Annu. Rev. Mater. Res. 49, 327 (2019).

As for the future, materials engineers are not on their own in the quest for weight reduction; numerous consortia with government, industrial, and academic partners have come into being in recent years to fast track the development. One is Lightweight Innovations for Tomorrow (LIFT), operated by the American Lightweight Materials Manufacturing Innovation Institute, a public-private partnership to develop and deploy advanced lightweight materials manufacturing technologies. Another is IACME, the composites institute. Comparable groups exist in Europe and Asia, most notably the Fraunhofer Institutes in Germany. Not only do these consortia provide a vehicle for information sharing and collaboration forming, some such as the Lightweight Materials (LightMAt) consortium provide access to advanced facilities at DOE national laboratories.

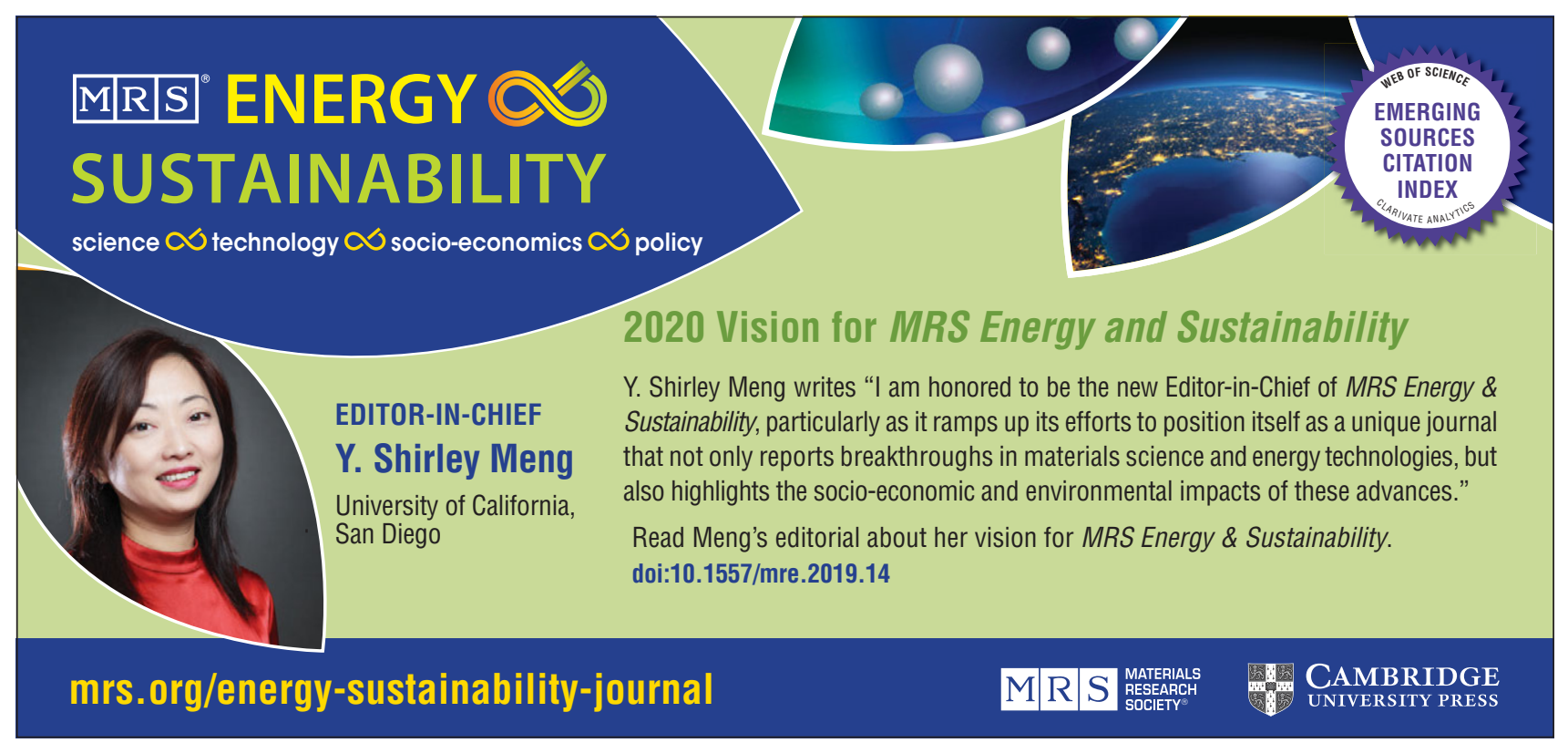




\section{MIRIs' ENERGY @ SUSTAINABILITY \\ science $\propto$ technology $\propto$ socio-economics $\propto$ policy}
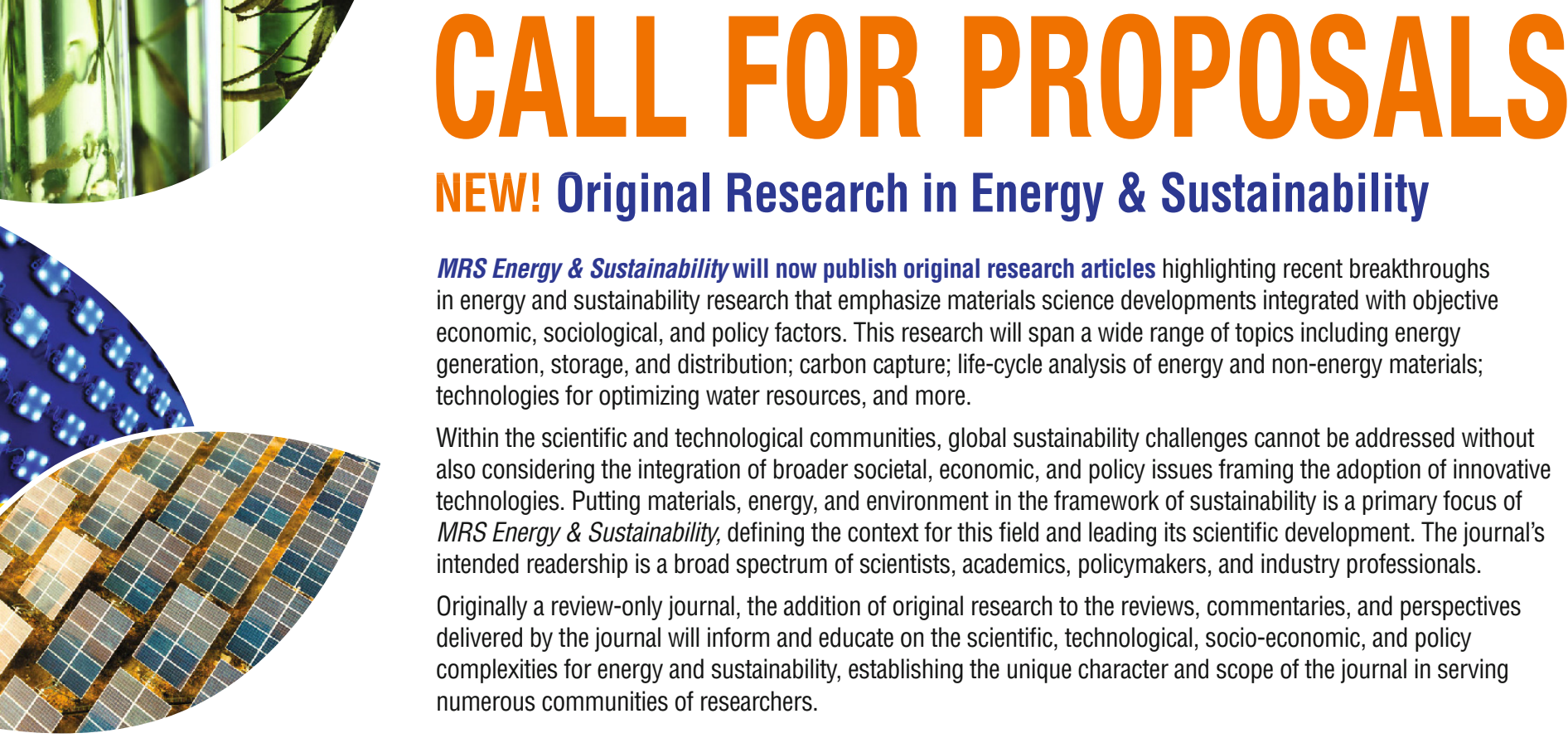

\section{NEW! Original Research in Energy \& Sustainability}

MRS Energy \& Sustainability will now publish original research articles highlighting recent breakthroughs in energy and sustainability research that emphasize materials science developments integrated with objective economic, sociological, and policy factors. This research will span a wide range of topics including energy generation, storage, and distribution; carbon capture; life-cycle analysis of energy and non-energy materials; technologies for optimizing water resources, and more.

Within the scientific and technological communities, global sustainability challenges cannot be addressed without also considering the integration of broader societal, economic, and policy issues framing the adoption of innovative technologies. Putting materials, energy, and environment in the framework of sustainability is a primary focus of MRS Energy \& Sustainability, defining the context for this field and leading its scientific development. The journal's intended readership is a broad spectrum of scientists, academics, policymakers, and industry professionals.

Originally a review-only journal, the addition of original research to the reviews, commentaries, and perspectives delivered by the journal will inform and educate on the scientific, technological, socio-economic, and policy complexities for energy and sustainability, establishing the unique character and scope of the journal in serving numerous communities of researchers.

Proposals for original research papers are solicited in the following areas, including but not limited to:
- Energy generation (solar, wind, and nuclear)
- Energy storage (batteries, biofuels, solar fuels, supercapacitors)
- New forms of energy distribution and usage enabled by these new materials (such as future electronics, neuromorphic devices, sensors, etc.)
- Electrocatalysis and photocatalysis
- Materials for carbon capture and storage
- Life-cycle analysis (LCA) of new energy materials and systems
- Life-cycle analysis for applications other than energy (electronics, plastics)
- Reducing or making substitution for use of rare or toxic materials
- Designing materials properties for long life or transience
- Use of plastics in the environment
- Artificial intelligence to speed research for sustainability solutions
- Synthetic biology for materials development
- Technologies for water purification or conversion

\section{Submission of Proposals}

To be considered, proposals outlining new but complete and previously unpublished results significant to the development of this field should be submitted via the MRS Energy \& Sustainability electronic submission system. The proposal form and author instructions may be found at mrs. org/energy-sustainability-proposal-form.

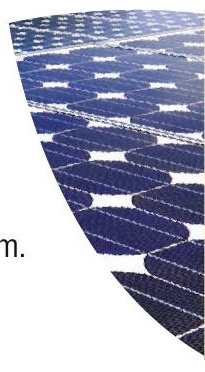

\section{Editor-in-Chief}

Y. Shirley Meng

University of California, San Diego 